This item was submitted to Loughborough's Research Repository by the author.

Items in Figshare are protected by copyright, with all rights reserved, unless otherwise indicated.

\title{
Electromagnetic guided waves on linear arrays of spheres
}

PLEASE CITE THE PUBLISHED VERSION

http://dx.doi.org/10.1016/j.wavemoti.2012.06.002

PUBLISHER

(c) Elsevier

VERSION

AM (Accepted Manuscript)

LICENCE

CC BY-NC-ND 4.0

REPOSITORY RECORD

Linton, C.M., V.V. Zalipaev, and lan Thompson. 2019. "Electromagnetic Guided Waves on Linear Arrays of Spheres". figshare. https://hdl.handle.net/2134/15303. 
This item was submitted to Loughborough's Institutional Repository (https://dspace.lboro.ac.uk/) by the author and is made available under the following Creative Commons Licence conditions.

\section{creative
commons}

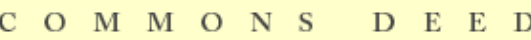

Attribution-NonCommercial-NoDerivs 2.5

You are free:

- to copy, distribute, display, and perform the work

Under the following conditions:

Attribution. You must attribute the work in the manner specified b the author or licensor.

Noncommercial. You may not use this work for commercial purposes.

No Derivative Works. You may not alter, transform, or build upon this work.

- For any reuse or distribution, you must make clear to others the license terms of this work.

- Any of these conditions can be waived if you get permission from the copyright holder.

Your fair use and other rights are in no way affected by the above.

This is a human-readable summary of the Leqal Code (the full license).

\section{Disclaimer 만}

For the full text of this licence, please go to: http://creativecommons.org/licenses/by-nc-nd/2.5/ 


\title{
Electromagnetic guided waves on linear arrays of spheres
}

\author{
C M Linton, V Zalipaev, and I Thompson, Department of Mathematical Sciences, \\ Loughborough University, Leicestershire, LE11 3TU, UK
}

\begin{abstract}
Guided electromagnetic waves propagating along one-dimensional arrays of dielectric spheres are studied. The quasi-periodic wave field is constructed as a superposition of vector spherical wave functions and then application of the boundary condition on the sphere surfaces leads to an infinite system of real linear algebraic equations. The vanishing of the determinant of the associated infinite matrix provides the condition for surface waves to exist and these are determined numerically after truncation of the infinite system. Dispersion curves are presented for a range of azimuthal modes and the effects of varying the sphere radius and electric permitivity are shown. We also demonstrate that a suitable truncation of the full system is precisely equivalent to the dipole approximation that has been used previously by other authors, in which the incident field on a sphere is approximated by its value at the centre of that sphere.
\end{abstract}

\section{Introduction}

Guided modes propagating along periodic structures have received considerable attention and, depending on the physical contexts, are known variously as edge waves [1], RayleighBloch surface waves [2], array-guided surface waves [3] or bound states [4]. Most of this work has focused on two-dimensional problems. There have been previous studies of electromagnetic surface waves guided by periodic arrays, but these have concentrated on cases where the spheres can be modelled by some combination of electric and magnetic dipoles $[5,6,7,8]$.

The work described here is a complete analysis, based on the full Maxwell equations, of travelling electromagnetic waves propagating along linear arrays of dielectric spheres in the absence of any incident field. A theory for electromagnetic wave scattering by an infinite planar array of spheres was developed in $[9,10]$ and our approach builds on the formalism presented there; it would be straightforward to modify our analysis to consider the corresponding scattering problem. A similar formalism for full vector problems in elasticity has also been developed $[11,12,13]$.

Our goal is to provide a thorough study of the modes that can exist. The problem is a natural extension of the equivalent acoustic problem [14] and, as in that case, there is a cut-off frequency below which waves cannot radiate energy away from the array. The modes that we seek have frequencies below this cut-off and decay exponentially as one moves away from the array. We do not address the far more difficult question of whether surface modes exist at frequencies above the cut-off (in which case they are usually referred to as embedded modes); a review of work on embedded modes in two dimensions can be found in [15].

In section 2 we formulate the general problem of electromagnetic waves traveling on an arbitrary infinite periodic array of penetrable dielectric spheres and then the special case of a one-dimensional array is treated in section 3. We derive a homogeneous infinite system of real linear algebraic equations and the condition for the existence of a guided wave is that the determinant of this system should vanish. The matrix associated with this system is truncated and the determinant then computed numerically; results are presented in section 4 . 


\section{Representation of the electromagnetic field}

We assume time-harmonic fields with an $\exp (-\mathrm{i} \omega t)(\omega>0)$ dependence throughout. Spherical polar coordinates are $(r, \theta, \phi)$ with unit vectors $\mathbf{e}_{r}, \mathbf{e}_{\theta}, \mathbf{e}_{\phi}$. In an isotropic homogeneous, source free medium Maxwell's equations are

$$
\begin{array}{rlrl}
\nabla \times \mathbf{E}-\mathrm{i} \omega \mu \mathbf{H} & =0, & \nabla \cdot \mathbf{E}=0, \\
\nabla \times \mathbf{H}+\mathrm{i} \omega \epsilon \mathbf{E}=0, & \nabla \cdot \mathbf{H}=0 .
\end{array}
$$

Here $\mathbf{E}$ is the electric intensity, $\mathbf{H}$ is the magnetic intensity, $\epsilon$ is the electric permitivity, and $\mu$ is the magnetic permeability. It follows that both $\mathbf{E}$ and $\mathbf{H}$ satisfy the vector Helmholtz equation:

$$
\nabla^{2} \mathbf{E}+k^{2} \mathbf{E}=0, \quad \nabla^{2} \mathbf{H}+k^{2} \mathbf{H}=0,
$$

in which $k^{2}=\omega^{2} \epsilon \mu$. Our analysis is built around vector spherical wavefunctions $\mathbf{M}_{n}^{m}$ and $\mathbf{N}_{n}^{m}$, defined in Appendix A, which are divergence-free solutions to the vector Helmholtz equation. Vector spherical wavefunctions which are regular at the origin (i.e. with the function $\mathrm{z}_{n}(k r)$ in Appendix A taken as the spherical Bessel function of the first kind, $\mathrm{j}_{n}(k r)$ ) will be denoted by $\hat{\mathbf{M}}_{n}^{m}$ and $\hat{\mathbf{N}}_{n}^{m}$ and we will use the notation $\mathbf{M}_{n}^{m}$ and $\mathbf{N}_{n}^{m}$ for functions containing spherical Hankel functions $\mathrm{h}_{n}^{(1)}(k r)$ (which are singular at the origin and behave like outgoing waves as $k r \rightarrow \infty)$.

To begin with we will consider an arbitrary periodic array $\Lambda$ of dielectric spheres, centred at the points $\mathbf{R}_{j} \in \Lambda$. Without loss of generality we can assume that $\mathbf{R}_{0}=\mathbf{0}$. Outside the spheres we write $\epsilon$ and $\mu$ for the electric permitivity and the magnetic permeability, respectively, whereas inside the spheres we use $\epsilon^{\prime}$ and $\mu^{\prime}$ with $k^{\prime}=\omega \sqrt{\epsilon^{\prime} \mu^{\prime}}$. We seek a quasi-periodic solution which satisfies

$$
\mathbf{E}\left(\mathbf{r}+\mathbf{R}_{j}\right)=\mathrm{e}^{\mathrm{i} \boldsymbol{\beta} \cdot \mathbf{R}_{j}} \mathbf{E}(\mathbf{r}), \quad \mathbf{H}\left(\mathbf{r}+\mathbf{R}_{j}\right)=\mathrm{e}^{\mathrm{i} \boldsymbol{\beta} \cdot \mathbf{R}_{j}} \mathbf{H}(\mathbf{r})
$$

for some Bloch vector $\boldsymbol{\beta}$. Let

$$
\mathbf{r}_{j}=\mathbf{r}-\mathbf{R}_{j} .
$$

Assuming that there is no incident field, we can then represent the electric and magnetic field outside the spheres via

$$
\begin{aligned}
& \mathbf{E}(\mathbf{r})=\sum_{\mathbf{R}_{j} \in \Lambda} \mathrm{e}^{\mathrm{i} \boldsymbol{\beta} \cdot \mathbf{R}_{j}} \sum_{n, m}\left(a_{n}^{m} \mathbf{M}_{n}^{m}\left(\mathbf{r}_{j}\right)+b_{n}^{m} \mathbf{N}_{n}^{m}\left(\mathbf{r}_{j}\right)\right), \\
& \mathbf{H}(\mathbf{r})=-\mathrm{i} \zeta \sum_{\mathbf{R}_{j} \in \Lambda} \mathrm{e}^{\mathrm{i} \boldsymbol{\beta} \cdot \mathbf{R}_{j}} \sum_{n, m}\left(a_{n}^{m} \mathbf{N}_{n}^{m}\left(\mathbf{r}_{j}\right)+b_{n}^{m} \mathbf{M}_{n}^{m}\left(\mathbf{r}_{j}\right)\right),
\end{aligned}
$$

where the complex coefficients $a_{n}^{m}$ and $b_{n}^{m}$ are to be determined, $\zeta=\sqrt{\epsilon / \mu}$ is the admittance of the medium and we have introduced the shorthand notation

$$
\sum_{n, m} \equiv \sum_{n=1}^{\infty} \sum_{m=-n}^{n} .
$$

Note that, unlike in the equivalent scalar problem, there is no monopole $(n=m=0)$ term. Spherically symmetric solutions to the source-free Maxwell equations exist only in the static 
case $k \rightarrow 0$ (see $[16, \S 9.1]$ ). Inside sphere $j$ the field is given by

$$
\begin{aligned}
\hat{\mathbf{E}}_{j}\left(\mathbf{r}_{j}\right) & =\mathrm{e}^{\mathrm{i} \boldsymbol{\beta} \cdot \mathbf{R}_{j}} \sum_{n, m}\left(a_{n}^{\prime m} \hat{\mathbf{M}}_{n}^{\prime m}\left(\mathbf{r}_{j}\right)+b_{n}^{\prime m} \hat{\mathbf{N}}_{n}^{\prime m}\left(\mathbf{r}_{j}\right)\right), \\
\hat{\mathbf{H}}_{j}\left(\mathbf{r}_{j}\right) & =-\mathrm{i} \zeta^{\prime} \mathrm{e}^{\mathrm{i} \boldsymbol{\beta} \cdot \mathbf{R}_{j}} \sum_{n, m}\left(a_{n}^{\prime m} \hat{\mathbf{N}}_{n}^{\prime m}\left(\mathbf{r}_{j}\right)+b_{n}^{\prime m} \hat{\mathbf{M}}_{n}^{\prime m}\left(\mathbf{r}_{j}\right)\right) .
\end{aligned}
$$

in which the primed quantities $\zeta^{\prime}, \hat{\mathbf{M}}_{n}^{\prime m}$ and $\hat{\mathbf{N}}_{n}^{\prime m}$ are the same as their unprimed equivalents except with $\epsilon$ and $\mu$ replaced with $\epsilon^{\prime}$ and $\mu^{\prime}$ appropriate for the medium inside the spheres.

It then suffices to consider the boundary conditions on a single sphere, which we take to be that at the origin. We can separate the total field, given by (6) and (7), into parts incident on and radiating from this particular sphere; the latter is given by

$$
\begin{aligned}
\mathbf{E}_{0}^{\mathrm{rad}}(\mathbf{r}) & =\sum_{n, m}\left(a_{n}^{m} \mathbf{M}_{n}^{m}(\mathbf{r})+b_{n}^{m} \mathbf{N}_{n}^{m}(\mathbf{r})\right), \\
\mathbf{H}_{0}^{\mathrm{rad}}(\mathbf{r}) & =-\mathrm{i} \zeta \sum_{n, m}\left(a_{n}^{m} \mathbf{N}_{n}^{m}(\mathbf{r})+b_{n}^{m} \mathbf{M}_{n}^{m}(\mathbf{r})\right) .
\end{aligned}
$$

The field incident on the sphere at the origin (which is made up from the radiated fields from all the other spheres) can be expanded, in the vicinity of that sphere, in terms of regular wavefunctions:

$$
\begin{aligned}
& \mathbf{E}_{0}^{\mathrm{inc}}(\mathbf{r})=\sum_{n, m}\left(c_{n}^{m} \hat{\mathbf{M}}_{n}^{m}(\mathbf{r})+d_{n}^{m} \hat{\mathbf{N}}_{n}^{m}(\mathbf{r})\right), \\
& \mathbf{H}_{0}^{\mathrm{inc}}(\mathbf{r})=-\mathrm{i} \zeta \sum_{n, m}\left(c_{n}^{m} \hat{\mathbf{N}}_{n}^{m}(\mathbf{r})+d_{n}^{m} \hat{\mathbf{M}}_{n}^{m}(\mathbf{r})\right),
\end{aligned}
$$

and then comparing this with (6) and (7) and using the addition theorem (72) we find that

$$
c_{\nu}^{\mu}=\sum_{n, m}\left(a_{n}^{m} \mathcal{A}_{n \nu}^{m \mu}+b_{n}^{m} \mathcal{B}_{n \nu}^{m \mu}\right), \quad d_{\nu}^{\mu}=\sum_{n, m}\left(a_{n}^{m} \mathcal{B}_{n \nu}^{m \mu}+b_{n}^{m} \mathcal{A}_{n \nu}^{m \mu}\right),
$$

where we have defined the lattice sums

$$
\mathcal{A}_{n \nu}^{m \mu}=\sum_{\mathbf{R}_{j} \in \Lambda}^{\prime} \mathrm{e}^{\mathrm{i} \boldsymbol{\beta} \cdot \mathbf{R}_{j}} A_{n \nu}^{m \mu}\left(-\mathbf{R}_{j}\right), \quad \mathcal{B}_{n \nu}^{m \mu}=\sum_{\mathbf{R}_{j} \in \Lambda}^{\prime} \mathrm{e}^{\mathrm{i} \boldsymbol{\beta} \cdot \mathbf{R}_{j}} B_{n \nu}^{m \mu}\left(-\mathbf{R}_{j}\right),
$$

with $A_{n \nu}^{m \mu}(\cdot)$ and $B_{n \nu}^{m \mu}(\cdot)$ given by (76) and (79), respectively. Here the dash on the summation indicates that the $j=0$ term is to be omitted.

The boundary conditions to be satisfied at $r=a$ are that the tangential component of both $\mathbf{E}$ and $\mathbf{H}$ must be continuous. In other words $\mathbf{e}_{r} \times \mathbf{E}$ and $\mathbf{e}_{r} \times \mathbf{H}$ need to be continuous. Using vector spherical harmonics defined by (58), and (63), (66) and the fact that $\mathbf{e}_{r} \times\left(\mathbf{e}_{r} \times \mathbf{X}_{n}^{m}\right)=-\mathbf{X}_{n}^{m}$, we see that continuity of $\mathbf{e}_{r} \times \mathbf{E}$ implies that

$$
\begin{aligned}
\sum_{n, m}\left(a_{n}^{\prime m} \mathrm{j}_{n}\right. & \left.\left(k^{\prime} a\right) \mathbf{e}_{r} \times \mathbf{X}_{n}^{m}-b_{n}^{\prime m} \mathrm{Dj}_{n}\left(k^{\prime} a\right) \mathbf{X}_{n}^{m}\right) \\
& =\sum_{n, m}\left(\left(a_{n}^{m} \mathrm{~h}_{n}(k a)+c_{n}^{m} \mathrm{j}_{n}(k a)\right) \mathbf{e}_{r} \times \mathbf{X}_{n}^{m}-\left(b_{n}^{m} \mathrm{D} \mathrm{h}_{n}(k a)+d_{n}^{m} \mathrm{D}_{n}(k a)\right) \mathbf{X}_{n}^{m}\right)
\end{aligned}
$$


and continuity of $\mathbf{e}_{r} \times \mathbf{H}$ gives

$$
\begin{aligned}
& \zeta^{\prime} \sum_{n, m}\left(b_{n}^{\prime m} \mathrm{j}_{n}\left(k^{\prime} a\right) \mathbf{e}_{r} \times \mathbf{X}_{n}^{m}-a_{n}^{\prime m} \mathrm{Dj}_{n}\left(k^{\prime} a\right) \mathbf{X}_{n}^{m}\right) \\
& \quad=\zeta \sum_{n, m}\left(\left(b_{n}^{m} \mathrm{~h}_{n}(k a)+d_{n}^{m} \mathrm{j}_{n}(k a)\right) \mathbf{e}_{r} \times \mathbf{X}_{n}^{m}-\left(a_{n}^{m} \mathrm{D} \mathrm{h}_{n}(k a)+c_{n}^{m} \mathrm{D}_{n}(k a)\right) \mathbf{X}_{n}^{m}\right)
\end{aligned}
$$

Here $\mathrm{D}$ is the differential operator defined in (67).

Next we take the dot product of each of (16) and (17) with $\overline{\mathbf{X}_{p}^{q}}$ and integrate over the unit sphere to yield for each $p=0,1,2, \ldots$ and $q=-p, \ldots, p$, on account of (59) and (69),

$$
b_{p}^{\prime q} \mathrm{Dj}_{p}\left(k^{\prime} a\right)=b_{p}^{q} \mathrm{D} \mathrm{h}_{p}(k a)+d_{p}^{q} \mathrm{Dj}_{p}(k a)
$$

and

$$
\frac{\zeta^{\prime}}{\zeta} a_{p}^{\prime q} \mathrm{Dj}_{p}\left(k^{\prime} a\right)=a_{p}^{q} \mathrm{D} \mathrm{h}_{p}(k a)+c_{p}^{q} \mathrm{Dj}_{p}(k a)
$$

Similarly, if we take the cross product of (16) and (17) with $\overline{\mathbf{X}_{p}^{q}}$ and integrate over the unit sphere we obtain, on account of (61) and (62),

$$
a_{p}^{\prime q} \mathrm{j}_{p}\left(k^{\prime} a\right)=a_{p}^{q} \mathrm{~h}_{p}(k a)+c_{p}^{q} \mathrm{j}_{p}(k a)
$$

and

$$
\frac{\zeta^{\prime}}{\zeta} b_{p}^{\prime q} \mathrm{j}_{p}\left(k^{\prime} a\right)=b_{p}^{q} \mathrm{~h}_{p}(k a)+d_{p}^{q} \mathrm{j}_{p}(k a) .
$$

Elimination of the interior coefficients $a_{p}^{\prime q}$ and $b_{p}^{q q}$ leads to the relations

$$
a_{p}^{q}+Z_{p}^{(1)} c_{p}^{q}=0, \quad b_{p}^{q}+Z_{p}^{(2)} d_{p}^{q}=0
$$

where the so-called Lorenz-Mie coefficients $Z_{p}^{(i)}$ are given by

$$
\begin{aligned}
& Z_{p}^{(1)}=\frac{\zeta^{\prime} \mathrm{j}_{p}(k a) \mathrm{Dj}_{p}\left(k^{\prime} a\right)-\zeta \mathrm{D}_{p}(k a) \mathrm{j}_{p}\left(k^{\prime} a\right)}{\zeta^{\prime} \mathrm{h}_{p}(k a) \mathrm{D}_{p}\left(k^{\prime} a\right)-\zeta \mathrm{Dh}_{p}(k a) \mathrm{j}_{p}\left(k^{\prime} a\right)} \\
& Z_{p}^{(2)}=\frac{\zeta^{\prime} \mathrm{D} \mathrm{j}_{p}(k a) \mathrm{j}_{p}\left(k^{\prime} a\right)-\zeta \mathrm{j}_{p}(k a) \mathrm{Dj}_{p}\left(k^{\prime} a\right)}{\zeta^{\prime} \mathrm{Dh}_{p}(k a) \mathrm{j}_{p}\left(k^{\prime} a\right)-\zeta \mathrm{h}_{p}(k a) \mathrm{D}_{p}\left(k^{\prime} a\right)}
\end{aligned}
$$

(These are equivalent to the expressions given in [17, p. 565].) Finally, if we substitute from (14) we obtain the coupled systems of homogeneous equations

$$
\left.\begin{array}{r}
a_{p}^{q}+Z_{p}^{(1)} \sum_{n, m}\left(a_{n}^{m} \mathcal{A}_{n p}^{m q}+b_{n}^{m} \mathcal{B}_{n p}^{m q}\right)=0 \\
b_{p}^{q}+Z_{p}^{(2)} \sum_{n, m}\left(a_{n}^{m} \mathcal{B}_{n p}^{m q}+b_{n}^{m} \mathcal{A}_{n p}^{m q}\right)=0
\end{array}\right\} \quad p \geq 1,|q| \leq p
$$

For the purpose of making numerical computations we truncate this system. The crudest such truncation is to insist that $p=q=1$ (i.e. retain only the dipole terms). It is possible (as in [5]) to restrict attention at the outset to fields consisting solely of dipoles and then to replace the complicated machinery that results from the application of the addition theorem and which leads to (14) by the much simpler process of matching the value of the incident 
field at the centre of the chosen sphere. It is not obvious that this approach is equivalent to truncating (25) but this is the case, as we now demonstrate.

From (6), (7) we have, for dipole fields,

$$
\begin{aligned}
& \mathbf{E}_{0}^{\mathrm{inc}}(\mathbf{0})=\sum_{\mathbf{R}_{j} \in \Lambda}^{\prime} \mathrm{e}^{\mathrm{i} \boldsymbol{\beta} \cdot \mathbf{R}_{j}} \sum_{m=-1}^{1}\left(a_{1}^{m} \mathbf{M}_{1}^{m}\left(-\mathbf{R}_{j}\right)+b_{1}^{m} \mathbf{N}_{1}^{m}\left(-\mathbf{R}_{j}\right)\right), \\
& \mathbf{H}_{0}^{\mathrm{inc}}(\mathbf{0})=-\mathrm{i} \zeta \sum_{\mathbf{R}_{j} \in \Lambda}^{\prime} \mathrm{e}^{\mathrm{i} \boldsymbol{\beta} \cdot \mathbf{R}_{j}} \sum_{m=-1}^{1}\left(a_{1}^{m} \mathbf{N}_{1}^{m}\left(-\mathbf{R}_{j}\right)+b_{1}^{m} \mathbf{M}_{1}^{m}\left(-\mathbf{R}_{j}\right)\right),
\end{aligned}
$$

and the key step is to note that, from (85),

$$
\mathbf{M}_{1}^{m}\left(-\mathbf{R}_{j}\right)=\sum_{\mu=-1}^{1} B_{11}^{m \mu}\left(-\mathbf{R}_{j}\right) \hat{\mathbf{N}}_{1}^{\mu}(\mathbf{0}), \quad \mathbf{N}_{1}^{m}\left(-\mathbf{R}_{j}\right)=\sum_{\mu=-1}^{1} A_{11}^{m \mu}\left(-\mathbf{R}_{j}\right) \hat{\mathbf{N}}_{1}^{\mu}(\mathbf{0})
$$

On the other hand, from (12) and (13), using (70), we have

$$
\mathbf{E}_{0}^{\mathrm{inc}}(\mathbf{0})=\sum_{m=-1}^{1} d_{1}^{m} \hat{\mathbf{N}}_{1}^{m}(\mathbf{0}), \quad \mathbf{H}_{0}^{\mathrm{inc}}(\mathbf{0})=-\mathrm{i} \zeta \sum_{m=-1}^{1} c_{1}^{m} \hat{\mathbf{N}}_{1}^{m}(\mathbf{0}) .
$$

Matching (29) with (26) and (27), using (15), thus yields

$$
\begin{aligned}
& \sum_{\mu=-1}^{1} d_{1}^{\mu} \hat{\mathbf{N}}_{1}^{\mu}(\mathbf{0})=\sum_{m=-1}^{1} \sum_{\mu=-1}^{1}\left(a_{1}^{m} \mathcal{B}_{11}^{m \mu}+b_{1}^{m} \mathcal{A}_{11}^{m \mu}\right) \hat{\mathbf{N}}_{1}^{\mu}(\mathbf{0}), \\
& \sum_{\mu=-1}^{1} c_{1}^{\mu} \hat{\mathbf{N}}_{1}^{\mu}(\mathbf{0})=\sum_{m=-1}^{1} \sum_{\mu=-1}^{1}\left(a_{1}^{m} \mathcal{A}_{11}^{m \mu}+b_{1}^{m} \mathcal{B}_{11}^{m \mu}\right) \hat{\mathbf{N}}_{1}^{\mu}(\mathbf{0}),
\end{aligned}
$$

and this is clearly satisfied if

$$
c_{1}^{\mu}=\sum_{m=-1}^{1}\left(a_{1}^{m} \mathcal{A}_{11}^{m \mu}+b_{1}^{m} \mathcal{B}_{11}^{m \mu}\right), \quad d_{1}^{\mu}=\sum_{m=-1}^{1}\left(a_{1}^{m} \mathcal{B}_{11}^{m \mu}+b_{1}^{m} \mathcal{A}_{11}^{m \mu}\right), \quad \mu=-1,0,1
$$

which is the truncated version of (14). In the general case this is a $6 \times 6$ system of equations.

\section{Guided waves on linear arrays of spheres}

The system of equations (25) applies equally well to a one-, two-, or three-dimensional lattice $\Lambda$. In this section we consider the special case of a linear array of spheres, i.e. we take

$$
\mathbf{R}_{j}=j s \mathbf{e}_{z}, \quad j \in \mathbb{Z},
$$

where $s$ is the spacing between the sphere centres. In this case the modes corresponding to different values of $m$ decouple, since if $m \neq q$, then $\mathcal{A}_{n p}^{m q}=\mathcal{B}_{n p}^{m q}=0$ (see Appendix B) and without loss of generality we can assume that $m \geq 0$. We take $\boldsymbol{\beta}=\beta \mathbf{e}_{z}$ and since $\exp \left(\mathrm{i} \boldsymbol{\beta} \cdot \mathbf{R}_{j}\right)=\exp (\mathrm{i} j s \beta)$ it is clear that we only need to consider $\beta s \in(-\pi, \pi]$. However, 
the symmetry of the geometry means that in fact we only need to consider $\beta s \in[0, \pi]$. Then outside the spheres we have the representations

$$
\begin{aligned}
& \mathbf{E}_{m}(\mathbf{r})=\sum_{j=-\infty}^{\infty} \mathrm{e}^{\mathrm{i} j s \beta} \sum_{n=m^{*}}^{\infty}\left(a_{n}^{m} \mathbf{M}_{n}^{m}\left(\mathbf{r}_{j}\right)+b_{n}^{m} \mathbf{N}_{n}^{m}\left(\mathbf{r}_{j}\right)\right) \\
& \mathbf{H}_{m}(\mathbf{r})=-\mathrm{i} \zeta \sum_{j=-\infty}^{\infty} \mathrm{e}^{\mathrm{i} j s \beta} \sum_{n=m^{*}}^{\infty}\left(a_{n}^{m} \mathbf{N}_{n}^{m}\left(\mathbf{r}_{j}\right)+b_{n}^{m} \mathbf{M}_{n}^{m}\left(\mathbf{r}_{j}\right)\right),
\end{aligned}
$$

where we have written $m^{*}=\max (1, m)$. We have, from (83) and (84),

$$
\begin{gathered}
\mathcal{A}_{n \nu}^{m m}=4 \pi(-1)^{m} \sqrt{\frac{\nu(\nu+1)}{n(n+1)}} \sum_{\substack{p=|n-\nu| \\
n+\nu+p \text { even }}}^{n+\nu} \mathrm{i}^{\nu-n-p} g_{n \nu p} \mathcal{G}(n, m ; \nu,-m ; p) \sigma_{p}, \\
\mathcal{B}_{n \nu}^{m m}=\frac{2 \pi(-1)^{m}}{\sqrt{n(n+1) \nu(\nu+1)}} \sum_{\substack{p=|n-\nu|+1 \\
n+\nu+p \text { odd }}}^{n+\nu-1} \mathrm{i}^{\nu-n-p} \sqrt{\frac{2 p+1}{2 p-1}} \mathcal{H}(n, m ; \nu,-m ; p) \sigma_{p},
\end{gathered}
$$

where $\sigma_{p}$ is defined in (86).

For a particular value of $m$, the system of equations given by (25) reduces to

$$
\left.\begin{array}{r}
a_{\nu}^{m}+Z_{\nu}^{(1)} \sum_{n=m^{*}}^{\infty}\left(a_{n}^{m} \mathcal{A}_{n \nu}^{m m}+b_{n}^{m} \mathcal{B}_{n \nu}^{m m}\right)=0 \\
b_{\nu}^{m}+Z_{\nu}^{(2)} \sum_{n=m^{*}}^{\infty}\left(a_{n}^{m} \mathcal{B}_{n \nu}^{m m}+b_{n}^{m} \mathcal{A}_{n \nu}^{m m}\right)=0
\end{array}\right\} \nu \geq m^{*}
$$

When $0<k s<\beta s<\pi$, which ensures no radiation away from the array, this system of complex equations can be transformed into a real system as follows. We use (75), (78), (93) and (94), to show that

$$
\mathcal{A}_{n \nu}^{m m}=\mathrm{i}^{\nu-n+1} \tilde{\mathcal{A}}_{n \nu}^{m}-\delta_{n \nu}, \quad \mathcal{B}_{n \nu}^{m m}=\mathrm{i}^{\nu-n+1} \tilde{\mathcal{B}}_{n \nu}^{m},
$$

where

$$
\begin{gathered}
\tilde{\mathcal{A}}_{n \nu}^{m}=4 \pi(-1)^{m} \sqrt{\frac{\nu(\nu+1)}{n(n+1)}} \sum_{\substack{p=|n-\nu| \\
n+\nu+p \text { even }}}^{n+\nu} g_{n \nu p} \mathcal{G}(n, m ; \nu,-m ; p)(-1)^{p} \eta_{p}, \\
\tilde{\mathcal{B}}_{n \nu}^{m}=\frac{2 \pi(-1)^{m}}{\sqrt{n(n+1) \nu(\nu+1)}} \sum_{\substack{p=|n-\nu|+1 \\
n+\nu+p \text { odd }}}^{n+\nu-1} \sqrt{\frac{2 p+1}{2 p-1}} \mathcal{H}(n, m ; \nu,-m ; p)(-1)^{p} \eta_{p}
\end{gathered}
$$

and $\eta_{p}$ is defined in (94). Thus (38) becomes

$$
\left.\begin{array}{l}
\mathrm{i}^{-\nu} a_{\nu}^{m}+W_{\nu}^{(1)} \sum_{n=m^{*}}^{\infty} \mathrm{i}^{-n}\left(a_{n}^{m} \tilde{\mathcal{A}}_{n \nu}^{m}+b_{n}^{m} \tilde{\mathcal{B}}_{n \nu}^{m}\right)=0 \\
\mathrm{i}^{-\nu} b_{\nu}^{m}+W_{\nu}^{(2)} \sum_{n=m^{*}}^{\infty} \mathrm{i}^{-n}\left(a_{n}^{m} \tilde{\mathcal{B}}_{n \nu}^{m}+b_{n}^{m} \tilde{\mathcal{A}}_{n \nu}^{m}\right)=0
\end{array}\right\} \nu \geq m^{*}
$$


where

$$
W_{\nu}^{(i)}=\frac{\mathrm{i} Z_{\nu}^{(i)}}{1-Z_{\nu}^{(i)}}, \quad i=1,2,
$$

which depend on the product of the wavenumber $k$ and the spheres radius $a$. The coefficients $\tilde{\mathcal{A}}_{n \nu}^{m}, \tilde{\mathcal{B}}_{n \nu}^{m}$ depend only on the two non-dimensional parameters $k s$ and $\beta s$. Substituting the definitions of the Lorenz-Mie coefficients (23), (24) shows that $W_{\nu}^{(i)}$ is real and so (42) is a real system with unknowns $\mathrm{i}^{-\nu} a_{\nu}^{m}$ and $\mathrm{i}^{-\nu} b_{\nu}^{m}$.

The existence of guided waves along the array corresponds to values of $k$ and $\beta$ for which this system admits non-trivial solutions for the unknowns $a_{n}^{m}, b_{n}^{m}$. Thus, equating to zero the determinant of the system we come to dispersion relations connecting $k$ and $\beta$.

The special case $m=1$, and $a_{n}^{1}=b_{n}^{1}=0$ for $n>1$, is worthy of consideration since this corresponds to the dipole approximation considered in $[5,18]$. (In fact, those papers consider a particular polarization which is a linear combination of the $m=1$ and $m=-1$ modes, but the dispersion relation is identical.) The general $6 \times 6$ system given in (32) reduces to a $2 \times 2$ system in this case. From (42), dropping the superfluous sub- and superscripts $n, \nu$ and $m$ (which are all 1 ) we get the (real) dispersion relation

$$
\left(1+W^{(1)} \tilde{\mathcal{A}}\right)\left(1+W^{(2)} \tilde{\mathcal{A}}\right)-W^{(1)} W^{(2)} \tilde{\mathcal{B}}^{2}=0
$$

and $\tilde{\mathcal{A}}$ and $\tilde{\mathcal{B}}$ can be determined using (40), (41), (75), (78), together with the results

$$
\mathcal{G}(1,1 ; 1,-1 ; 2)=1 / \sqrt{20 \pi}, \quad \mathcal{H}(1,1 ; 1,-1,1)=-1 / \sqrt{\pi} .
$$

We obtain

$$
\tilde{\mathcal{A}}=\sqrt{4 \pi} \eta_{0}+\sqrt{\frac{\pi}{5}} \eta_{2}, \quad \tilde{\mathcal{B}}=-\sqrt{3 \pi} \eta_{1}
$$

For convenience, we define

$$
\chi_{q}^{ \pm}=\left(\mathrm{Cl}_{q}(k s+\beta s) \pm \mathrm{Cl}_{q}(k s-\beta s)\right) /(k s)^{q} .
$$

Then, using (94), we have

$$
\tilde{\mathcal{A}}=-\frac{3}{2}\left(\chi_{1}^{+}-\chi_{2}^{+}-\chi_{3}^{+}\right), \quad \tilde{\mathcal{B}}=\frac{3}{2}\left(\chi_{1}^{-}-\chi_{2}^{-}\right) .
$$

To allow easy comparison with [5], we re-write the dispersion relation (44), using (43), as

$$
\frac{\mathrm{i} Z^{(1)} \tilde{\mathcal{B}}}{1+Z^{(1)}(\mathrm{i} \tilde{\mathcal{A}}-1)}=\frac{1+Z^{(2)}(\mathrm{i} \tilde{\mathcal{A}}-1)}{\mathrm{i} Z^{(2)} \tilde{\mathcal{B}}}
$$

and define $\Sigma_{1,2}$ and $S_{ \pm}$via

$$
\Sigma_{1}=\chi_{1}^{+}-\chi_{2}^{+}-\chi_{3}^{+}-\frac{2 \mathrm{i}}{3}, \quad \Sigma_{2}=\chi_{1}^{-}-\chi_{2}^{-}, \quad S_{+}=3 \mathrm{i} Z^{(1)} / 2, \quad S_{-}=3 \mathrm{i} Z^{(2)} / 2 .
$$

Then (49) becomes

$$
\frac{S_{+} \Sigma_{2}}{1-S_{+} \Sigma_{1}}=\frac{1-S_{-} \Sigma_{1}}{S_{-} \Sigma_{2}}
$$

which is equivalent to equation (18) of [5]. 
Table 1: Comparison of values of $\beta$ for $2.715<k<2.750$ and $m=0, a=0.47$ and $\epsilon^{\prime}=10$ given for $N=1,2,4,8$

\begin{tabular}{|c|c|c|c|c|}
\hline$k$ & $\beta, 2 \times 2$ & $\beta, 4 \times 4$ & $\beta, 8 \times 8$ & $\beta, 16 \times 16$ \\
\hline 2.715 & 2.718 & 2.843 & 2.897 & 2.899 \\
2.720 & 2.733 & 2.859 & 2.917 & 2.920 \\
2.725 & 2.750 & 2.875 & 2.940 & 2.942 \\
2.730 & 2.770 & 2.893 & 2.965 & 2.967 \\
2.735 & 2.793 & 2.912 & 2.994 & 2.997 \\
2.740 & 2.820 & 2.933 & 3.031 & 3.034 \\
2.745 & 2.851 & 2.956 & 3.063 & 3.059 \\
2.750 & 2.888 & 2.927 & 2.869 & 2.866 \\
\hline
\end{tabular}

\section{Numerical results}

Results are presented with the problem scaled so that the spacing between consecutive sphere centres is unity (i.e. $s=1$ ). To restrict the number of parameters in the problem we also set $\epsilon=\mu=\mu^{\prime}=1$. This leaves three key parameters: the sphere radius $a$, the electric permitivity of the spheres $\epsilon^{\prime}$, and the azimuthal mode number $m$. Extensive numerical searches for guided waves with azimuthal mode numbers $m=0, \ldots, 4$ that can propagate along linear arrays were carried out using program code written in Fortran 2003. The Gaunt coefficients were computed using an updated version of the 'Root-Rational-Fraction package' described in [19] and the lattice sums were evaluated using the method described in [20].

For computational purposes the infinite linear system (42) (which converges exponentially) is truncated to a $2 N \times 2 N$ system. The choice of $N$ is then a balance between speed of computation and required accuracy. As the results which are presented below demonstrate, in most cases the crudest of truncations $(N=1)$ yields accurate results. The most challenging situation is when $a$ is large (the spheres touch when $a=0.5$ ) and $m=0$ and to illustrate the convergence as $N$ is increased in such a situation we present in Table 1 results of computed values of $\beta \in(k, \pi)$ for various values of $k$ in the interval $2.715<k<2.75$ with the parameters $m=0, a=0.47$ and $\epsilon^{\prime}=10$ for $N=1,2,4,8$, i.e. with system sizes $2 \times 2$, $4 \times 4,8 \times 8$ and $16 \times 16$. Even in this case, it is clear that small truncation parameters still yield extremely accurate results.

If the spheres are too small then we are unable to find any modes. In the absence of an existence proof for these guided waves (unlike in the equivalent acoustic problem where such proofs have recently been provided [21]) it is not possible to say whether this is because they do not exist or whether it is just that the values of $k$ and $\beta$ are so close that we are unable to resolve the difference. For $\epsilon^{\prime}=10$, the minimum values of $a$ for which modes have been computed are, approximately, $a=0.327$ for $m=0, a=0.205$ for $m=1$, and $a=0.463$ for $m=2$. We have been unable to find any solutions for $m>2$. It is not surprising, from a physical perspective, that it is more challenging to find modes for the linear array of spheres than in similar two-dimensional problems such as those mentioned in the first paragraph of the introduction. This is because the waves have an extra dimension in which to leak energy to infinity.

In Figure 1 we present the dispersion curves showing how $\beta$ varies with $k$ for $s=1$, $\epsilon=\mu=\mu^{\prime}=1$ and $\epsilon^{\prime}=10$. Continuous and dashed curves are for $a=0.4$ and $a=0.47$, respectively, computed using a truncation parameter of $N=8$, with the stars showing the results computed using $N=1$. Figure 1 (a) shows axisymmetric modes $(m=0)$ while 

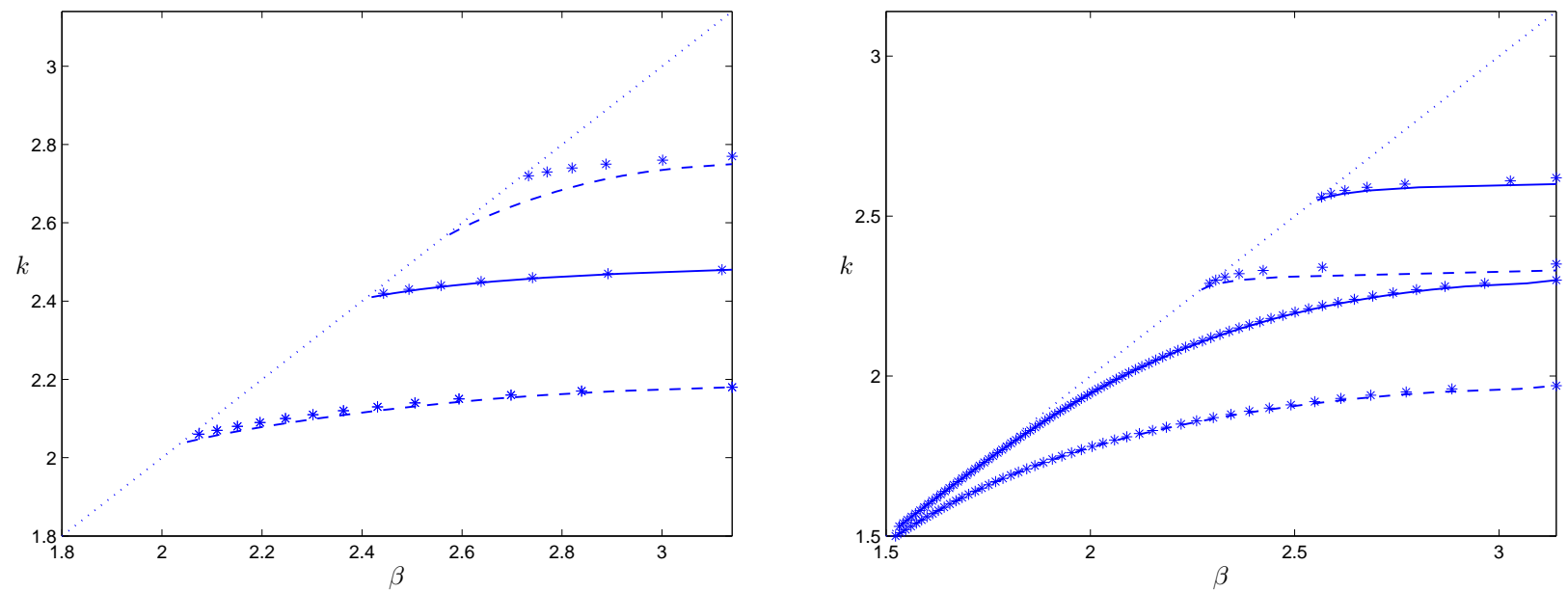

Figure 1: Dispersion curves for $s=1, \epsilon=\mu=\mu^{\prime}=1$ and $\epsilon^{\prime}=10$, computed using $N=8$. The dotted diagonal line corresponds to $\beta=k$. Solid and dashed curves are for $a=0.4$ and $a=0.47$, respectively. In (a) $m=0$, whereas in (b) $m=1$. In all cases the stars correspond to results computed using $N=1$.

Figure 1(b) is for $m=1$. Results for $a=0.4, m=1, N=1$ are given in Figure 17 of [18] and these agree with the stars approximating the solid curves in Figure 1(b). Results for $m=2$ (but with the other parameters as in Figure 1) are shown in Figure 2, this time for $a=0.47$ and $a=0.49$ as it is only for these large spheres that we have been able to find modes.

In Figure 3 we illustrate the effect of varying $\epsilon^{\prime}$ with $s=1, \epsilon=\mu=\mu^{\prime}=1$. The most obvious effect is that if $\epsilon^{\prime}$ is reduced sufficiently, modes cease to exist. As an example, Figure 3(a) shows results for $a=0.49$ and $m=0$, with different curves corresponding to, reading upwards, $\epsilon^{\prime}=4.3,4.2,4.1,4.0$ and 3.9. For $\epsilon^{\prime}=3.8$ we do not find any modes. On the other hand, increasing $\epsilon^{\prime}=20$ leads to the presence of more modes, as illustrated in Figure 3(b) which shows results for $\epsilon^{\prime}=20, a=0.4$ with the dashed curve for $m=0$ and the solid curve for $m=1$.

\section{Conclusion}

Dispersion curves describing guided electromagnetic waves propagating along one-dimensional arrays of dielectric spheres have been determined based on a full vector solution of Maxwell's equations. By writing the quasi-periodic wave field as a superposition of vector spherical wave functions and then applying the boundary condition on the sphere surfaces we are able to reduce the problem to an infinite system of real linear algebraic equations and surface waves exist when the determinant of the associated infinite matrix vanishes. A truncation procedure, with excellent convergence characteristics, has been used to enable these to be determined numerically.

Previous work based on a dipole approximation has shown that such guided waves do indeed exist. In this paper we have extended the understanding of these modes in two ways. First, we have demonstrated that the dipole approximation is equivalent to a truncation of the linear system that we derive from the full Maxwell equations and that the approximation yields accurate solutions over a wide parameter range so that it can be used with confidence. However, its accuracy diminishes as the sphere radius gets larger. This is because the higher modes are excited more strongly for larger spheres and also that more modes are important 


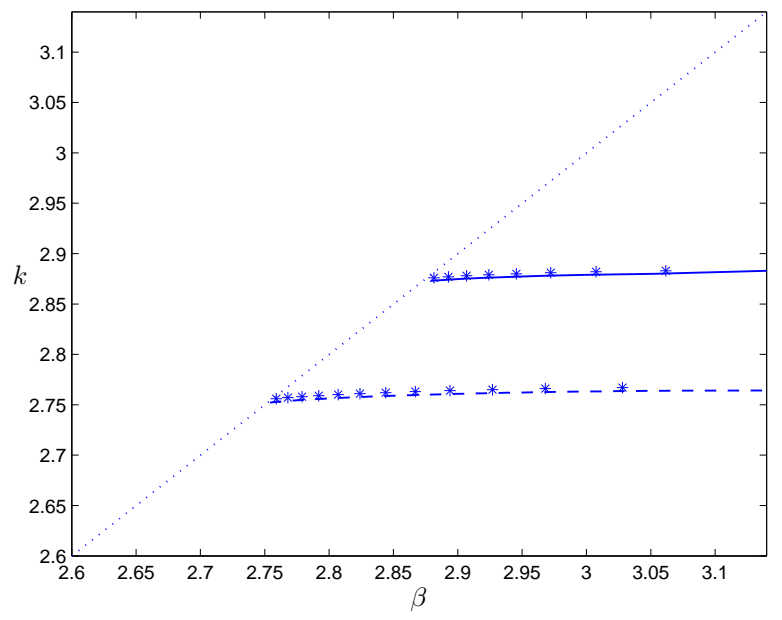

Figure 2: Dispersion curves for $s=1, \epsilon=\mu=\mu^{\prime}=10$ and $m=2$, computed using $N=8$. The dotted diagonal line corresponds to $\beta=k$. The solid curve is for $a=0.47$ while the dashed curve is for $a=0.49$. In both cases the stars correspond to results computed using $N=1$.

in the interaction theory when the space between the spheres reduces. Secondly we have shown that modes exist for azimuthal modes $|m|=0,1,2$ (previously only the case $|m|=1$ had been treated) but our computations suggest that there are no modes for $|m|>2$.

\section{A Vector spherical harmonics}

Spherical harmonics are defined by

$$
\mathrm{Y}_{n}^{m}\left(\mathbf{e}_{r}\right) \equiv \mathrm{Y}_{n}^{m}(\theta, \phi)=(-1)^{m} \lambda_{n m} \mathrm{P}_{n}^{m}(\cos \theta) \mathrm{e}^{\mathrm{i} m \phi}, \quad n \geq|m| \geq 0
$$

where

$$
\lambda_{n m}=\sqrt{\frac{(2 n+1)(n-m) !}{4 \pi(n+m) !}}
$$

and the associated Legendre function is defined here, for non-negative order and $|x| \leq 1$, by

$$
\mathrm{P}_{n}^{m}(x)=\left(1-x^{2}\right)^{m / 2} \frac{\mathrm{d}^{m}}{\mathrm{~d} x^{m}} \mathrm{P}_{n}(x)=\frac{\left(1-x^{2}\right)^{m / 2}}{2^{n} n !} \frac{\mathrm{d}^{m+n}}{\mathrm{~d} x^{m+n}}\left(x^{2}-1\right)^{n}, \quad n \geq m \geq 0 .
$$

This is the convention adopted in [22]. If $m>n, \mathrm{P}_{n}^{m}(x) \equiv 0$. The extension to negative order is accomplished via

$$
\mathrm{P}_{n}^{-m}(x)=(-1)^{m} \frac{(n-m) !}{(n+m) !} \mathrm{P}_{n}^{m}(x), \quad n \geq|m|
$$

We note that

$$
\overline{\mathrm{Y}_{n}^{m}\left(\mathbf{e}_{r}\right)}=(-1)^{m} \mathrm{Y}_{n}^{-m}\left(\mathbf{e}_{r}\right), \quad \mathrm{Y}_{n}^{m}\left(-\mathbf{e}_{r}\right)=(-1)^{n} \mathrm{Y}_{n}^{m}\left(\mathbf{e}_{r}\right)
$$

and

$$
\int_{\Omega} \mathrm{Y}_{n}^{m} \overline{\mathrm{Y}_{\nu}^{\mu}} \mathrm{d} \Omega=\delta_{n \nu} \delta_{m \mu}
$$

the integral being over the surface of the unit sphere. 

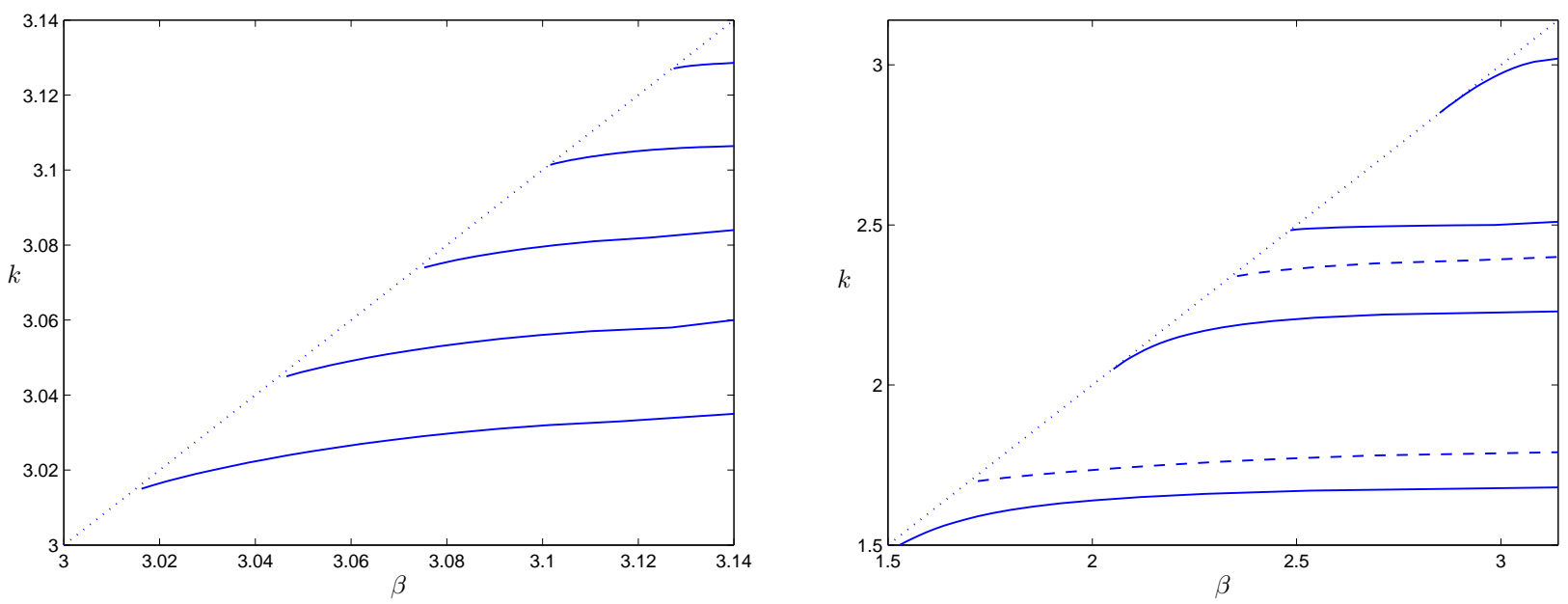

Figure 3: Dispersion curves for $s=1, \epsilon=\mu=\mu^{\prime}=1$. computed using $N=8$. The dotted diagonal line corresponds to $\beta=k$. In (a) $a=0.49$ and $m=0$ and the different curves are, from bottom to top, for $\epsilon^{\prime}=4.3,4.2,4.1,4.0$ and 3.9. In (b) $\epsilon^{\prime}=20, a=0.4$ with the dashed curve for $m=0$ and the solid curve for $m=1$.

For $n \geq 1$, we can define vector spherical harmonics by

$$
\mathbf{X}_{n}^{m}(\theta, \phi)=\frac{\nabla \mathrm{Y}_{n}^{m} \times \mathbf{r}}{\sqrt{n(n+1)}}=\frac{(-1)^{m} \lambda_{n m} \mathrm{e}^{\mathrm{i} m \phi}}{\sqrt{n(n+1)}}\left(\frac{\mathrm{i} m}{\sin \theta} \mathrm{P}_{n}^{m}(\cos \theta) \mathbf{e}_{\theta}-\partial_{\theta}\left(\mathrm{P}_{n}^{m}(\cos \theta)\right) \mathbf{e}_{\phi}\right)
$$

and it can be shown that

$$
\int_{\Omega} \mathbf{X}_{n}^{m} \cdot \overline{\mathbf{X}_{p}^{q}} \mathrm{~d} \Omega=\delta_{n p} \delta_{m q}
$$

(Note that no complex conjugation is implicit in the dot product.) Since $\overline{\mathbf{X}_{p}^{q}} \cdot \mathbf{e}_{r}=0$, we have

$$
\left(\mathbf{e}_{r} \times \mathbf{X}_{n}^{m}\right) \times \overline{\mathbf{X}_{p}^{q}}=-\left(\mathbf{X}_{n}^{m} \cdot \overline{\mathbf{X}_{p}^{q}}\right) \mathbf{e}_{r}
$$

and hence

$$
\int_{\Omega}\left(\mathbf{e}_{r} \times \mathbf{X}_{n}^{m}\right) \times \overline{\mathbf{X}_{p}^{q}} \mathrm{~d} \Omega=-\delta_{n p} \delta_{m q} \mathbf{e}_{r}
$$

We also have

$$
\int_{\Omega} \mathbf{X}_{n}^{m} \times \overline{\mathbf{X}_{p}^{q}} \mathrm{~d} \Omega=0
$$

Our first set of divergence free solutions of the Helmholtz equation are taken as

$$
\mathbf{M}_{n}^{m}=\mathrm{z}_{n}(k r) \mathbf{X}_{n}^{m}(\theta, \phi)
$$

and we have

$$
\int_{\Omega} \mathbf{M}_{n}^{m} \cdot \overline{\mathbf{X}_{p}^{q}} \mathrm{~d} \Omega=\delta_{n p} \delta_{m q} \mathrm{z}_{n}(k r) .
$$

The second set of divergence free solutions of the Helmholtz equation is

$$
\begin{aligned}
\mathbf{N}_{n}^{m} & =\frac{1}{k} \nabla \times \mathbf{M}_{n}^{m}=\frac{1}{k}\left(\mathrm{z}_{n}(k r) \nabla \times \mathbf{X}_{n}^{m}-\mathbf{X}_{n}^{m} \times \nabla \mathrm{z}_{n}(k r)\right) \\
& =\frac{\mathrm{z}_{n}(k r)}{k r} \sqrt{n(n+1)} \mathrm{Y}_{n}^{m} \mathbf{e}_{r}+\mathrm{D} \mathrm{z}_{n}(k r) \mathbf{e}_{r} \times \mathbf{X}_{n}^{m}
\end{aligned}
$$


where we have introduced the differential operator

$$
\mathrm{D} f(x)=\frac{1}{x} \frac{\mathrm{d}}{\mathrm{d} x}(x f(x)) .
$$

From the last of these we see that

$$
\mathbf{N}_{n}^{m} \cdot \overline{\mathbf{X}_{p}^{q}}=\mathrm{Dz}_{n}(k r) \mathbf{e}_{r} \times \mathbf{X}_{n}^{m} \cdot \overline{\mathbf{X}_{p}^{q}}
$$

and we can show that

$$
\int_{\Omega} \mathbf{N}_{n}^{m} \cdot \overline{\mathbf{X}_{p}^{q}} \mathrm{~d} \Omega=0
$$

For more details, see [17, Chapter 7] and [16, §9.7].

Note that

$$
\hat{\mathbf{M}}_{n}^{m}(\mathbf{0})=0, \quad \hat{\mathbf{N}}_{n}^{m}(\mathbf{0})=\frac{\delta_{n 1}}{3}\left(\sqrt{2} \mathrm{Y}_{n}^{m}(\theta, \phi) \mathbf{e}_{r}+2 \mathbf{e}_{r} \times \mathbf{X}_{n}^{m}\right) .
$$

If, for convenience, we introduce Cartesian unit vectors $\mathbf{e}_{x}, \mathbf{e}_{y}, \mathbf{e}_{z}$ then we have

$$
\hat{\mathbf{N}}_{1}^{0}(\mathbf{0})=\frac{\mathbf{e}_{z}}{\sqrt{6 \pi}}, \quad \hat{\mathbf{N}}_{1}^{ \pm 1}(\mathbf{0})=\frac{1}{\sqrt{12 \pi}}\left(\mp \mathbf{e}_{x}-\mathbf{i} \mathbf{e}_{y}\right)
$$

\section{B Addition theorems}

The addition theorem for vector spherical harmonics $[23,24]$ that we need here takes the form

$$
\left.\begin{array}{l}
\mathbf{M}_{n}^{m}(\mathbf{c})=\sum_{\nu, \mu}\left(A_{n \nu}^{m \mu}(\mathbf{b}) \hat{\mathbf{M}}_{\nu}^{\mu}(\mathbf{a})+B_{n \nu}^{m \mu}(\mathbf{b}) \hat{\mathbf{N}}_{\nu}^{\mu}(\mathbf{a})\right) \\
\mathbf{N}_{n}^{m}(\mathbf{c})=\sum_{\nu, \mu}\left(A_{n \nu}^{m \mu}(\mathbf{b}) \hat{\mathbf{N}}_{\nu}^{\mu}(\mathbf{a})+B_{n \nu}^{m \mu}(\mathbf{b}) \hat{\mathbf{M}}_{\nu}^{\mu}(\mathbf{a})\right)
\end{array}\right\},
$$

where $\mathbf{c}=\mathbf{a}+\mathbf{b}$ and $|\mathbf{a}|<|\mathbf{b}|$. Expressions for the $A_{n \nu}^{m \mu}(\mathbf{b})$ and $B_{n \nu}^{m \mu}(\mathbf{b})$ are given in [24], though care needs to be taken when converting his formulas to our notation, which is based on that of [22]. The formulas make use of so-called Gaunt coefficients, $\mathcal{G}$, which are integrals of products of three spherical harmonics:

$$
\mathcal{G}(n, m ; \nu, \mu ; p)=\int_{\Omega} \mathrm{Y}_{n}^{m} \mathrm{Y}_{\nu}^{\mu} \overline{\mathrm{Y}_{p}^{m+\mu}} \mathrm{d} \Omega
$$

the integration being over the surface of the unit sphere. These coefficients can also be expressed in terms of Wigner $3-j$ symbols via

$$
\mathcal{G}(n, m ; \nu, \mu ; p)=\frac{(-1)^{m+\mu}}{\sqrt{4 \pi}} \sqrt{(2 n+1)(2 \nu+1)(2 p+1)}\left(\begin{array}{ccc}
n & \nu & p \\
m & \mu & -m-\mu
\end{array}\right)\left(\begin{array}{lll}
n & \nu & p \\
0 & 0 & 0
\end{array}\right) .
$$

For future reference we note that

$$
\mathcal{G}(n, m ; \nu,-m ; 0)=\frac{(-1)^{m} \delta_{n \nu}}{\sqrt{4 \pi}}
$$

Theorems I and II of [24] imply that

$$
A_{n \nu}^{m \mu}(\mathbf{b})=4 \pi(-1)^{\mu} \mathrm{i}^{\nu-n} \sqrt{\frac{\nu(\nu+1)}{n(n+1)}} \sum_{\substack{p=|n-\nu| \\ n+\nu+p \text { even }}}^{n+\nu} g_{n \nu p} \mathrm{i}^{p} \mathcal{G}(n, m ; \nu,-\mu ; p) \mathrm{h}_{p}(k b) \mathrm{Y}_{p}^{m-\mu}(\hat{\mathbf{b}}),
$$


where $b=|\mathbf{b}|, \hat{\mathbf{b}}=\mathbf{b} / b$ and

$$
g_{n \nu p}=1+\frac{(n-\nu+p+1)(n+\nu-p)}{2 \nu(2 \nu+1)}-\frac{(\nu-n+p+1)(\nu+n+p+2)}{2(\nu+1)(2 \nu+1)} .
$$

For future reference we note that

$$
g_{n n 0}=1, \quad g_{112}=-1 / 2 .
$$

Similarly (note that there is a well-documented sign error in Cruzan's paper [25, 26, 27])

$$
B_{n \nu}^{m \mu}(\mathbf{b})=\frac{2 \pi(-1)^{\mu} \mathrm{i}^{\nu-n}}{\sqrt{n(n+1) \nu(\nu+1)}} \sum_{\substack{p=|n-\nu|+1 \\ n+\nu+p \text { odd }}}^{n+\nu-1} \mathrm{i}^{p} \sqrt{\frac{2 p+1}{2 p-1}} \mathcal{H}(n, m ; \nu,-\mu ; p) \mathrm{h}_{p}(k b) \mathrm{Y}_{p}^{m-\mu}(\hat{\mathbf{b}})
$$

where

$$
\mathcal{H}(n, m ; \nu, \mu ; p)=\sum_{j=-1}^{1} \mathcal{G}_{j}(n, m ; \nu, \mu ; p)
$$

with

$$
\begin{aligned}
\mathcal{G}_{0}(n, m ; \nu, \mu ; p)=- & 2 \mu \sqrt{p^{2}-(m+\mu)^{2}} \mathcal{G}(n, m ; \nu, \mu ; p-1), \\
\mathcal{G}_{ \pm 1}(n, m ; \nu, \mu ; p)=\mp & \sqrt{(\nu \mp \mu)(\nu \pm \mu+1)(p \mp(m+\mu))(p-1 \mp(m+\mu))} \\
& \times \mathcal{G}(n, m ; \nu, \mu \pm 1 ; p-1) .
\end{aligned}
$$

For the case of a linear array we have $\mathbf{a}=\mathbf{r}, \mathbf{b}=-\mathbf{R}_{j}=-j s \mathbf{e}_{z}$ and $\mathbf{c}=\mathbf{r}_{j}$. In this case $A_{n \nu}^{m \mu}\left(-\mathbf{R}_{j}\right)=B_{n \nu}^{m \mu}\left(-\mathbf{R}_{j}\right)=0$ if $m \neq \mu$, since $\mathrm{P}_{n}^{m}( \pm 1)=( \pm 1)^{n} \delta_{m 0}$. We then have

$$
\begin{aligned}
A_{n \nu}^{m m}\left(-\mathbf{R}_{j}\right)=4 \pi(-1)^{m} \mathrm{i}^{\nu-n} \sqrt{\frac{\nu(\nu+1)}{n(n+1)}} & \\
& \times \sum_{\substack{p=|n-\nu| \\
n+\nu+p \text { even }}}^{n+\nu} g_{n \nu p} \mathcal{G}(n, m ; \nu,-m ; p) \mathrm{h}_{p}(k|j s|) \lambda_{p 0}(-\mathrm{i} \operatorname{sgn} j)^{p}
\end{aligned}
$$

and

$$
\begin{aligned}
& B_{n \nu}^{m m}\left(-\mathbf{R}_{j}\right)=\frac{2 \pi(-1)^{m} \mathrm{i}^{\nu-n}}{\sqrt{n(n+1) \nu(\nu+1)}} \\
& \times \sum_{\substack{p=|n-\nu|+1 \\
n+\nu+p \text { odd }}}^{n+\nu-1} \sqrt{\frac{2 p+1}{2 p-1}} \mathcal{H}(n, m ; \nu,-m ; p) \mathrm{h}_{p}(k|j s|) \lambda_{p 0}(-\mathrm{i} \operatorname{sgn} j)^{p} .
\end{aligned}
$$

If we set $\mathbf{c}=\mathbf{r}, \mathbf{b}=\mathbf{r}$ and $\mathbf{a}=\mathbf{0}$ in (72) and use (70) we see that we must have

$$
\mathbf{M}_{n}^{m}(\mathbf{r})=\sum_{\mu=-1}^{1} B_{n 1}^{m \mu}(\mathbf{r}) \hat{\mathbf{N}}_{1}^{\mu}(\mathbf{0}), \quad \mathbf{N}_{n}^{m}(\mathbf{r})=\sum_{\mu=-1}^{1} A_{n 1}^{m \mu}(\mathbf{r}) \hat{\mathbf{N}}_{1}^{\mu}(\mathbf{0}) .
$$

These relations (which can, with difficulty, be verified) serve as a useful check on the expressions for $A_{n \nu}^{m \mu}$ and $B_{n \nu}^{m \mu}$. 


\section{Lattice sums}

We are concerned here with the lattice sums

$$
\sigma_{p}=\lambda_{p 0} \sum_{j=1}^{\infty} \mathrm{h}_{p}(k s j)\left(\mathrm{e}^{\mathrm{i} \beta s j}+(-1)^{p} \mathrm{e}^{-\mathrm{i} \beta s j}\right)
$$

with $\lambda_{p 0}$ defined in (53). We introduce the quantities

$$
\beta_{q}=\beta+2 q \pi / s, \quad q \in \mathbb{Z}
$$

and when $\left|\beta_{q}\right| \leq k$ we define $\psi_{q} \in[0, \pi]$ via $\cos \psi_{q}=\beta_{q} / k$. Provided there is no integer $q$ such that $\left|\beta_{q}\right|=k$, we then have (see [20] for the details)

$$
\begin{aligned}
\sigma_{p}=-\frac{\delta_{p 0}}{\sqrt{4 \pi}}+\frac{\pi \mathrm{i}^{p} \lambda_{p 0}}{k s} \sum_{\left|\beta_{q}\right|<k} \mathrm{P}_{p}\left(\cos \psi_{q}\right) \\
\quad+\lambda_{p 0}(-\mathrm{i})^{p+1} \sum_{q=0}^{p} \frac{c_{p q} \Omega_{q}}{(k s)^{q+1}}\left[\mathrm{Cl}_{q+1}(k s+\beta s)+(-1)^{p} \mathrm{Cl}_{q+1}(k s-\beta s)\right]
\end{aligned}
$$

where

$$
c_{p q}=\frac{(p+q) !}{2^{q} q !(p-q) !}, \quad \Omega_{q}= \begin{cases}\mathrm{i}^{q} & q \text { even, } \\ \mathrm{i}^{q+1} & q \text { odd },\end{cases}
$$

and $\mathrm{Cl}_{q}(\cdot)$ are the Clausen functions

$$
\mathrm{Cl}_{2 m}(x)=\sum_{j=1}^{\infty} \frac{\sin j x}{j^{2 m}}, \quad \mathrm{Cl}_{2 m+1}(x)=\sum_{j=1}^{\infty} \frac{\cos j x}{j^{2 m+1}} .
$$

The function $\mathrm{Cl}_{1}$ has the closed form

$$
\mathrm{Cl}_{1}(x)=-\frac{1}{2} \ln (2-2 \cos x)
$$

and as a result the lattice sum $\sigma_{0}$ has a particularly simple representation:

$$
\sigma_{0}=\frac{1}{k s \sqrt{4 \pi}}(M \pi-k s+\mathrm{i} \log [2|\cos \beta s-\cos k s|]),
$$

where $M$ is the number of integers $q$ for which $\left|\beta_{q}\right|<k$.

If $0<k s<\beta s<\pi$, then $\left|\beta_{q}\right|>k$ for all integers $q$. In this case we have

$$
\mathrm{i}^{p} \sigma_{p}=-\frac{\delta_{p 0}}{\sqrt{4 \pi}}+\mathrm{i} \eta_{p}
$$

where

$$
\eta_{p}=-\lambda_{p 0} \sum_{q=0}^{p} \frac{c_{p q} \Omega_{q}}{(k s)^{q+1}}\left[\mathrm{Cl}_{q+1}(k s+\beta s)+(-1)^{p} \mathrm{Cl}_{q+1}(k s-\beta s)\right]
$$

is real. We also have

$$
\eta_{0}=\frac{\log [2(\cos k s-\cos \beta s)]}{k s \sqrt{4 \pi}}
$$




\section{References}

[1] D. V. Evans and C. M. Linton. Edge waves along periodic coastlines. Q. J. Mech. Appl. Math., 46(4):642-656, 1993.

[2] R. Porter and D. V. Evans. Rayleigh-Bloch surface waves along periodic gratings and their connection with trapped modes in waveguides. J. Fluid Mech., 386:233-258, 1999.

[3] D. S. Janning and B. A. Munk. Effects of surface waves on the currents of truncated periodic arrays. IEEE Trans. Antennas Propagat., 50(9):1254-1265, 2002.

[4] J. Y. Vaishnav, J. D. Walls, M. Apratim, and E. J. Heller. Matter-wave scattering and guiding by atomic arrays. Phys. Rev. A, 76:013620 (13 pages), 2007.

[5] R. A. Shore and A. D. Yaghjian. Traveling electromagnetic waves on linear periodic arrays of lossless penetrable spheres. IEICE Trans. Commun., E88-B(6):2346-2352, 2005.

[6] A. Alù and N. Engheta. Theory of linear chains of metamaterial/plasmonic particles as subdiffraction optical nanotransmission lines. Phys. Rev. B, 74(20):205436 (18 pages), 2006.

[7] A. F. Koenderink and A. Polman. Complex response and polariton-like dispersion splitting in periodic metal nanoparticle chains. Phys. Rev. B, 74(3):033402 (4 pages), 2006.

[8] R. A. Shore and A. D. Yaghjian. Traveling waves on two- and three-dimensional periodic arrays of lossless scatterers. Radio Science, 42:RS6S21 (40 pages), 2007.

[9] A. Modinos. Scattering of electromagnetic waves by a plane of spheres - formalism. Physica A, 141:575-588, 1987.

[10] N. Stefanou and A. Modinos. Scattering of light from a two-dimensional array of spherical particles on a substrate. J. Phys.: Condens. Matter, 3:8135-8148, 1991.

[11] Z. Liu, C. T. Chan, P. Sheng, A. L. Goertzen, and J. H. Page. Elastic wave scattering by periodic structures of spherical objects: Theory and experiment. Phys. Rev. B, 62(4):2446-2457, 2000.

[12] I. E. Psarobas, N. Stefanou, A. Modinos, and V. Yannopapas and. Scattering of elastic waves by periodic arrays of spherical bodies. Phys. Rev. B, 62(1):278-291, 2000.

[13] R. Sainidou, N. Stefanou, I. E. Psarobas, and A. Modinos. Scattering of elastic waves by a periodic monolayer of spheres. Phys. Rev. B, 66:024303 (1-7), 2002.

[14] I. Thompson and C. M. Linton. Guided surface waves on one- and two-dimensional arrays of spheres. SIAM J. Appl. Math., 70(8):2975-2995, 2010.

[15] C. M. Linton and P. McIver. Embedded trapped modes in water waves and acoustics. Wave Motion, 45:16-29, 2007.

[16] J. D. Jackson. Classical Electrodynamics. John Wiley \& Sons, New York, 3rd edition, 1999. 
[17] J. A. Stratton. Electromagnetic Theory. McGraw-Hill, New York, 1941.

[18] R. A. Shore and A. D. Yaghjian. Traveling electromagnetic waves on linear periodic arrays of lossless penetrable spheres. Technical Report AFRL-SN-HS-TR-2004-044, Air Force Research Laboratory, 2004.

[19] A. J. Stone and C. P. Wood. Root-rational-fraction package for exact calculation of vector-coupling coefficients. Computer Physics Communications, 21:195-205, 1980.

[20] C. M. Linton and I. Thompson. One- and two-dimensional lattice sums for the threedimensional Helmholtz equation. J. Comput. Phys., 228(6):1815-1829, 2009.

[21] M. McIver. Acoustic wave trapping in one-dimensional axisymmetric arrays. Q. J. Mech. Appl. Math., 64(3):401-414, 2011.

[22] P. A. Martin. Multiple Scattering. Interaction of Time-Harmonic Waves with N Obstacles. Cambridge University Press, 2006.

[23] S. Stein. Addition theorems for spherical wave functions. Quart. Appl. Math., 19(1):15$24,1961$.

[24] O. R. Cruzan. Translational addition theorems for spherical vector wave functions. Quart. Appl. Math., 20(1):33-40, 1962.

[25] L. Tsang and J. A. Kong. Effective propagation constants for coherent electromagnetic wave propagation in media embedded with dielectric scatters. J. Appl. Phys., 53(11):7162-7173, 1982.

[26] P. C. Waterman and N. E. Pedersen. Electromagnetic scattering by periodic arrays of particles. J. Appl. Phys., 59(8):2609-2618, 1986.

[27] B. U. Felderhof and R. B. Jones. Addition theorems for spherical wave solutions of the vector Helmholtz equation. J. Math. Phys., 28(4):836-839, 1987. 